\title{
Portfolio management with
}

\section{cryptocurrencies: the role of estimation risk}

Article

Accepted Version

Creative Commons: Attribution-Noncommercial-No Derivative Works 4.0

Platanakis, E. and Urquhart, A. (2019) Portfolio management with cryptocurrencies: the role of estimation risk. Economics Letters, 177. pp. 76-80. ISSN 0165-1765 doi:

https://doi.org/10.1016/j.econlet.2019.01.019 Available at https://centaur.reading.ac.uk/81734/

It is advisable to refer to the publisher's version if you intend to cite from the work. See Guidance on citing.

To link to this article DOI: http://dx.doi.org/10.1016/j.econlet.2019.01.019

Publisher: Elsevier

All outputs in CentAUR are protected by Intellectual Property Rights law, including copyright law. Copyright and IPR is retained by the creators or other copyright holders. Terms and conditions for use of this material are defined in the End User Agreement.

$\underline{\text { www.reading.ac.uk/centaur }}$

\section{CentAUR}


Central Archive at the University of Reading

Reading's research outputs online 


\title{
Portfolio Management with Cryptocurrencies: The Role of Estimation Risk
}

\begin{abstract}
This paper contributes to the literature on cryptocurrencies, portfolio management and estimation risk by comparing the performance of naïve diversification, Markowitz diversification and the advanced Black-Litterman model with VBCs that controls for estimation errors in a portfolio of cryptocurrencies. We show that the advanced Black-Litterman model with VBCs yields superior out-of-sample risk-adjusted returns as well as lower risks. Our results are robust to the inclusion of transaction costs and short-selling, indicating that sophisticated portfolio techniques that control for estimation errors are preferred when managing cryptocurrency portfolios.
\end{abstract}

Keywords: Cryptocurrencies; Estimation Errors; Portfolio Optimization JEL: G1; G2; G11 


\section{Introduction}

The mean-variance portfolio optimization framework of Markowitz (1952) is highly sensitive to estimation errors in the input parameters, and this has been extensively documented in the literature (e.g. Kan and Zhou, 2007; Levy and Levy, 2014). Hence, several studies have investigated whether other naïve strategies (such as the $1 / \mathrm{N}$ ) can beat mean-variance optimal portfolio diversification and its extensions in the out-of-sample. For example, Board and Sutcliffe (1994) document that there is very little to select between $1 / \mathrm{N}$ and other more sophisticated estimation methods for portfolio selection, while DeMiguel et al (2009) show that the $1 / \mathrm{N}$ is superior to 14 different portfolio optimization models across a range of markets in the out-of-sample setting.

Interest in cryptocurrencies is growing, especially as an investment where Baur et al (2018) show that Bitcoin accounts are mainly used as a speculative investment and not as an alternative currency and medium of exchange. ${ }^{1}$ The diversification benefits of Bitcoin to other financial assets has been reported by Bouri et al (2017) and Corbet et al (2018a), while recently Kajtazi and Moro (2018) and Platanakis and Urquhart (2018) both report substantial benefits from including Bitcoin in traditional portfolios. Platanakis et al (2018a) show that there is very little to select between optimal mean-variance diversification and $1 / \mathrm{N}$ for a portfolio of cryptocurrencies. However, cryptocurrencies have been found to be highly volatile (Chaim and Laurini 2018) and therefore have higher potential estimation errors in their parameters that may make portfolio theory particularly problematic when applied to a portfolio of cryptocurrencies. In this paper, we attempt to highlight this issue and add to the debate of optimal versus naïve diversification in cryptocurrencies by applying a more advanced and sophisticated portfolio optimization technique. This technique uses alternative estimates for the input parameters and imposes tighter constraints to the weights of assets with higher potential estimation errors and we find that this technique outperforms both $1 / \mathrm{N}$ and the Markowitz portfolio optimization framework when applied to a portfolio of cryptocurrencies. Therefore our paper furthers the findings by Platanakis et al (2018a).

The rest of this paper is organized as follows. Section 2 presents the data and methodology, Section 3 contains the empirical results. We conclude in Section 4.

\footnotetext{
${ }^{1}$ For an up-to-date review of the literature of cryptocurrencies, see Corbet et al (2018b).
} 


\section{Data and Methodology}

\subsection{Data}

We collect weekly data on Bitcoin, Litecoin, Ripple and Dash over the period $21^{\text {st }}$ February 2014 to $4^{\text {th }}$ May 2018 (220 weekly observations in total) from www.coinmarketcap.com, as well as for the risk-free rate from the Kenneth French web-site. ${ }^{2}$ Correlations are reported in Table 1 where we find the highest correlation between Litecoin and Ripple to be 0.5588 , while the lowest correlation is between Dash and Ripple at 0.0294 .

\subsection{Methodology}

\subsubsection{1/N Model}

Initially, we employ the $1 / \mathrm{N}$ rule which does not require any optimization and assigns a portfolio weight of $1 / \mathrm{N}$ to each asset. We use $1 / \mathrm{N}$ with re-balancing as in DeMiguel et al (2009).

\subsubsection{Markowitz Model}

The mean-variance portfolio optimization framework of Markowitz can be viewed as the choice of portfolio weights $(\mathbf{x})$ that maximize the Sharpe ratio. We also impose additional constraints to prohibit short selling $\left(\mathrm{x}_{i} \geq 0\right)$, and for the normalization of portfolio weights $\left(\sum_{i=1}^{N} \mathrm{x}_{i}=1\right)$. The optimization problem is written as follows:-

$$
\begin{array}{ll}
\max _{\mathbf{x}}\left\{\frac{\mathbf{x}^{\mathrm{T}} \boldsymbol{\mu}-\bar{r}_{f}}{\sqrt{\mathbf{x}^{\mathrm{T}} \boldsymbol{\Sigma} \mathbf{x}}}\right\} & \\
\text { s.t. } & \mathrm{x}_{i} \geq 0, \quad \forall i \\
& \sum_{i=1}^{N} \mathrm{x}_{i}=1
\end{array}
$$

\footnotetext{
${ }^{2}$ Bitcoin, Litecoin, Ripple and Dash stand as the most liquid cryptocurrencies. The starting date has been determined by the availability of all the cryptocurrencies used in this study. We choose weekly data since monthly data would not provide enough observations for a robust analysis while daily prices would result in a large turnover and thus high transaction costs. The Kenneth French database can be found at http://mba.tuck.dartmouth.edu/pages/faculty/ken.french/data library.html.
} 
where the parameters $\boldsymbol{\mu}$ and $\boldsymbol{\Sigma}$ denote the sample estimates for the means and the covariance matrix respectively, while $\bar{r}_{f}$ represents the average risk-free rate over the estimation period.

\subsubsection{Black-Litterman with VBCs}

The Black-Litterman (BL) portfolio optimization approach is an alternative portfolio optimization framework for dealing with estimation risk which has recently gained great attention, see for instance Bessler et al (2017), Platanakis and Sutcliffe (2017) and Oikonomou et al (2018), amongst others. The BL technique combines the subjective estimates (views) on returns and the benchmark portfolio to compute the implied returns.

Black and Litterman (1992) compute the vector of implied excess-returns (H) as follows:-

$$
\mathbf{H}=\lambda \Sigma \mathbf{x}^{\text {Benchmark }} \text {. }
$$

The vector $\mathbf{x}^{\text {Benchmark }}$ represents the benchmark portfolio and $\lambda$ denotes the relative risk aversion ${ }^{3}$.

The column vector of mean returns $\left(\boldsymbol{\mu}_{\mathrm{BL}}\right)$ is computed as follows:-

$$
\boldsymbol{\mu}_{\mathrm{BL}}=\left[(c \boldsymbol{\Sigma})^{-1}+\boldsymbol{P}^{\mathrm{T}} \boldsymbol{\Omega}^{-1} \boldsymbol{P}\right]^{-1}\left[(c \boldsymbol{\Sigma})^{-1} \mathbf{H}+\boldsymbol{P}^{\mathrm{T}} \boldsymbol{\Omega}^{-1} \mathbf{Q}\right]
$$

where the column vector $\mathbf{Q}$ contains the subjective returns and $\boldsymbol{P}$ represents a binary matrix with only non-zero elements in its diagonal. We also follow Platanakis and Sutcliffe (2017) and set the parameter $c$, which represents the overall level of confidence in the implied asset returns, to 0.1625. Meucci (2010) defines the diagonal matrix $\boldsymbol{\Omega}$ as follows:-

$$
\boldsymbol{\Omega}=\frac{1}{\delta} \boldsymbol{P} \Sigma \boldsymbol{P}^{\mathrm{T}}
$$

$3 \mathbf{x}^{\text {Benchmark }}$ is set to the global minimum-variance portfolio as in Bessler et al (2017), and many others. Since we maximize the Sharpe ratio, the parameter $\lambda$ is set to unity. 
where $1 / \delta$ is set to unity as in Meucci (2010). We also follow Platanakis et al (2018B) and Bessler et al (2017) and use the mean returns in each estimation period for setting the subjective returns in $\boldsymbol{Q}$. The covariance matrix $\left(\boldsymbol{\Sigma}_{\mathrm{BL}}\right)$ is computed as follows (Satchell and Scowcroft, 2000):-

$$
\boldsymbol{\Sigma}_{\mathrm{BL}}=\boldsymbol{\Sigma}+\left[(c \boldsymbol{\Sigma})^{-1}+\boldsymbol{P}^{\mathrm{T}} \boldsymbol{\Omega}^{-1} \boldsymbol{P}\right]^{-1}
$$

We additionally impose variance-based constraints (VBCs) of Levy and Levy (2014) to control further the negative impact of estimation errors in the input parameters. VBCs impose tighter constraints on the weights of the assets with the higher potential estimation risk, and are described as follows:-

$$
\left|\mathrm{x}_{i}-\frac{1}{N}\right| \frac{\sigma_{l}}{\bar{\sigma}} \leq a, \quad \forall i
$$

where $\bar{\sigma}=\frac{1}{N} \sum_{i=1}^{N} \sigma_{i}$, and the threshold $a$ is set to $20 \%$.

Hence, the optimization model is expressed as follows:-

$$
\begin{array}{ll}
\max _{\mathbf{x}} & \left\{\frac{\mathbf{x}^{\mathrm{T}} \boldsymbol{\mu}_{\mathrm{BL}}-\bar{r}_{f}}{\sqrt{\mathbf{x}^{\mathrm{T}} \boldsymbol{\Sigma}_{\mathrm{BL}} \mathbf{x}}}\right\} \\
\text { s.t. } \quad & \mathrm{x}_{i} \geq 0, \quad \forall i \\
& \sum_{i=1}^{N} \mathrm{x}_{i}=1, \\
& \left|\mathrm{x}_{i}-\frac{1}{N}\right| \frac{N \sigma_{i}}{\sum_{i=1}^{N} \sigma_{i}} \leq a, \quad \forall i
\end{array}
$$

where $\boldsymbol{\mu}_{\mathrm{BL}}=\left[(c \boldsymbol{\Sigma})^{-1}+\boldsymbol{P}^{\mathrm{T}} \boldsymbol{\Omega}^{-1} \boldsymbol{P}\right]^{-1}\left[(c \boldsymbol{\Sigma})^{-1} \mathbf{H}+\boldsymbol{P}^{\mathrm{T}} \boldsymbol{\Omega}^{-1} \mathbf{Q}\right]$ and $\boldsymbol{\Sigma}_{\mathrm{BL}}=\boldsymbol{\Sigma}+\left[(c \boldsymbol{\Sigma})^{-1}+\boldsymbol{P}^{\mathrm{T}} \boldsymbol{\Omega}^{-1} \boldsymbol{P}\right]^{-1}$. 


\subsection{Transaction Costs}

The total transaction costs $\left(\mathrm{TC}_{t}\right)$ are estimated as follows:-

$$
\mathrm{TC}_{t}=\sum_{i=1}^{N} \mathrm{~T}_{i}\left(\left|\mathrm{x}_{i, t}-\mathrm{x}_{i, t-1}^{+}\right|\right),
$$

where $\mathrm{x}_{i, t-1}^{+}$represents the proportion of the asset $i$ at the end of the period $\mathrm{t}-1$. We set the proportionate transaction cost $\left(\mathrm{T}_{\mathrm{i}}\right)$ per transaction to $50 \mathrm{bps}$ for all cryptocurrencies as in Lintilhac and Tourin (2017). The total transaction costs are subtracted from portfolio returns when measuring performance.

\section{Empirical Results}

Figure 1 reports the out-of-sample and net of transaction costs annualized Sharpe ratio for the 1/N rule, the Markowitz mean-variance portfolio model and the Black-Litterman technique with VBCs by using a 110-week rolling estimation window (half of the entire sample period) and rebalanced every week. ${ }^{45}$ We observe that the Black-Litterman approach with VBCs outperforms the both the $1 / \mathrm{N}$ and Markowitz benchmarks indicating that the advanced portfolio optimization model offers higher risk-adjusted returns for a cryptocurrency portfolio, inclusive of transaction costs. In Figure 2 we report the standard deviation of the of each portfolio and apart from the first 3 months of the reported out-of-sample period, the Black-Litterman model has a lower portfolio risk than the $1 / \mathrm{N}$ and Markowitz models highlighting the risk reduction from the more advanced optimization technique. For robustness, in Figure 3 we present the re-estimation of the 3 portfolio models with a $100 \%$ increase in the transaction cost estimates (e.g. 100 bps rather than $50 \mathrm{bps}$ ) and again show that the Black-Litterman model offers higher Sharpe ratios throughout the out-of-sample period. Finally in Figure 4 we allow for short-selling and consistent with the previous findings, the more advanced portfolio optimization model offers higher risk-adjusted returns throughout the reported out-of-sample period.

\footnotetext{
4 All analysis is conducted in Matlab.

${ }^{5}$ We allow for some weeks (out-of-sample observations) until we start reporting the out-of-sample Sharpe ratio in each figure, since its estimation with just a few observations may cause instability problems.
} 


\section{Conclusions}

We contribute to the literature on cryptocurrencies and estimation risk management by highlighting the fact that portfolio theory may face significant difficulties when applied to a portfolio of cryptocurrencies given the higher potential estimation errors in their parameters. To this end, we use an advanced portfolio optimization methodology and show that the BlackLitterman model with VBCs yields superior out-of-sample performance than other traditional benchmarks when applied to a portfolio of cryptocurrencies. This indicates that investors should use more sophisticated portfolio techniques that control for estimation errors in the input parameters when managing cryptocurrency portfolios. 


\section{Tables}

Table 1: Correlation matrix of the returns of the cryptocurrencies employed in this study.

\begin{tabular}{ccccc}
\hline Correlation Matrix & Bitcoin & Litecoin & Ripple & Dash \\
\hline Bitcoin & 1.0000 & & & \\
Litecoin & 0.5202 & 1.0000 & & \\
Ripple & 0.2465 & 0.5588 & 1.0000 & 1.0000 \\
Dash & 0.1658 & 0.1415 & 0.0294 & \\
\hline
\end{tabular}




\section{Figures}

Figure 1: The out-of-sample Sharpe ratio over time of the advanced portfolio optimization model and the 2 benchmarks (1/N and Markowitz), inclusive of transaction costs, for a 110 -week rolling window. Short selling is prohibited.

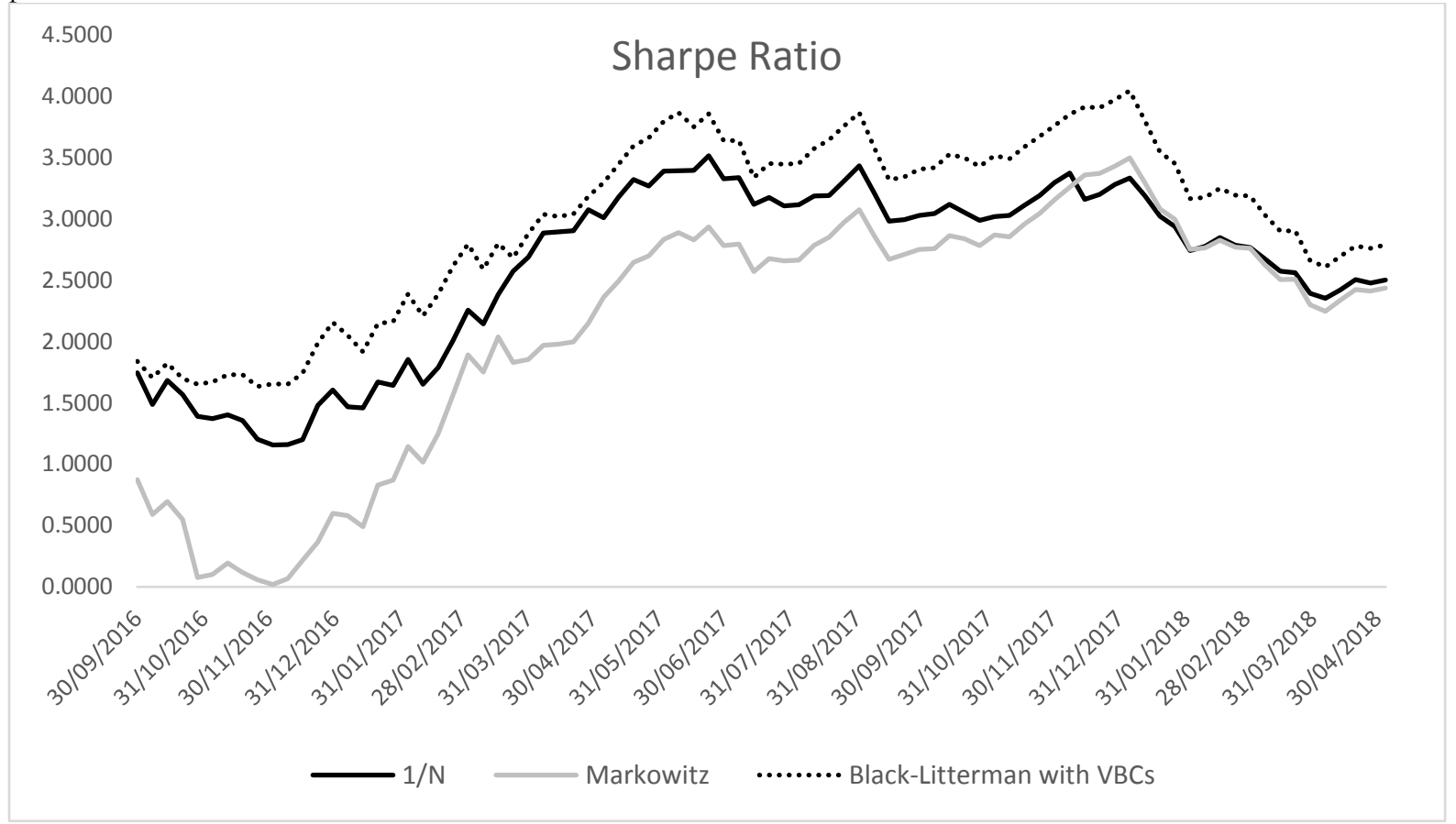

Figure 2: The out-of-sample portfolio standard deviation over time of the advanced portfolio optimization model and the 2 benchmarks (1/N and Markowitz), inclusive of transaction costs, for a 110 -week rolling window. Short selling is prohibited.

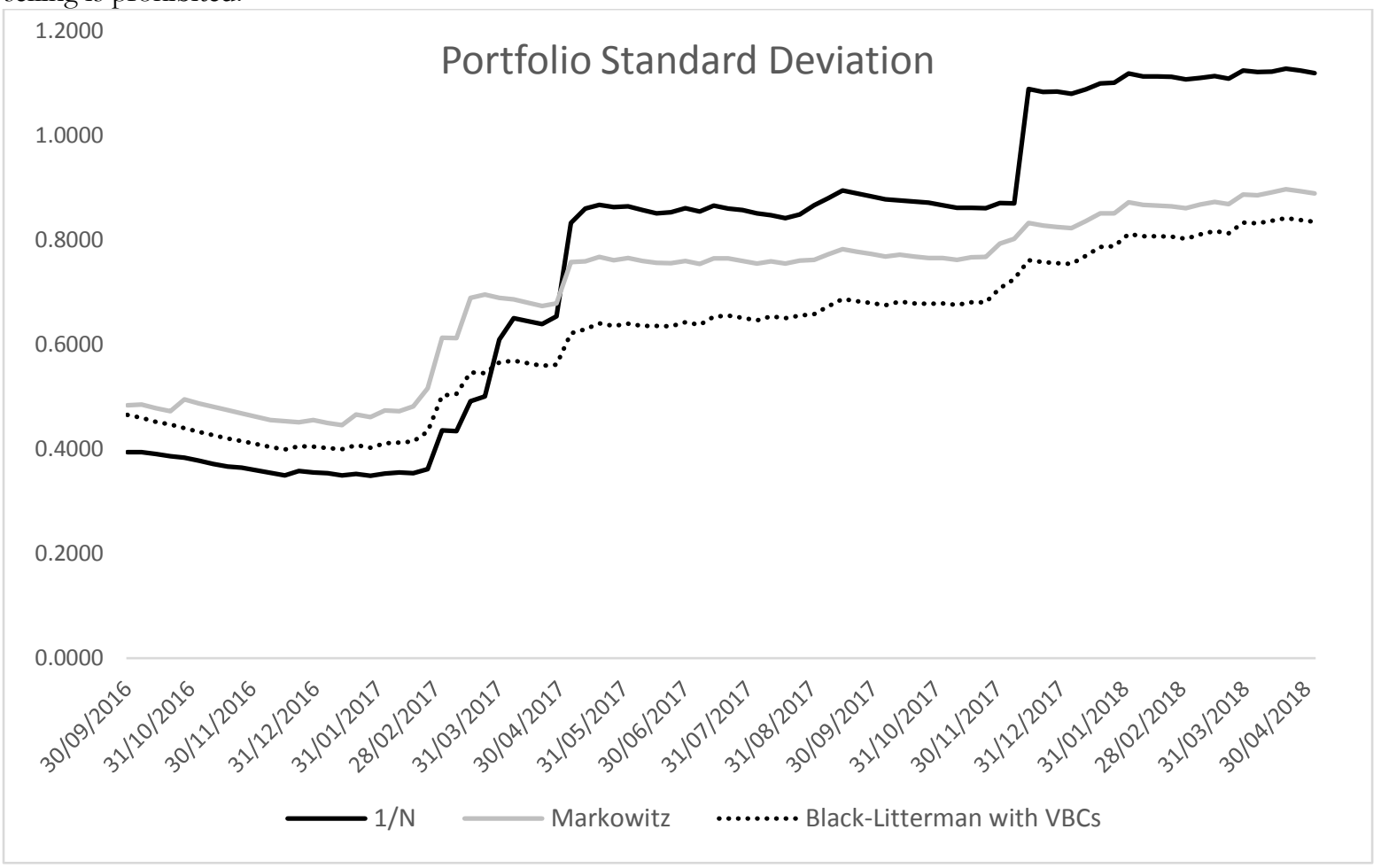


Figure 3: The out-of-sample Sharpe ratio over time of the advanced portfolio optimization model and the 2 benchmarks (1/N and Markowitz), for a 110-week rolling window. The proportional transaction cost estimates are set to $100 \mathrm{bps}(100 \%$ increase).

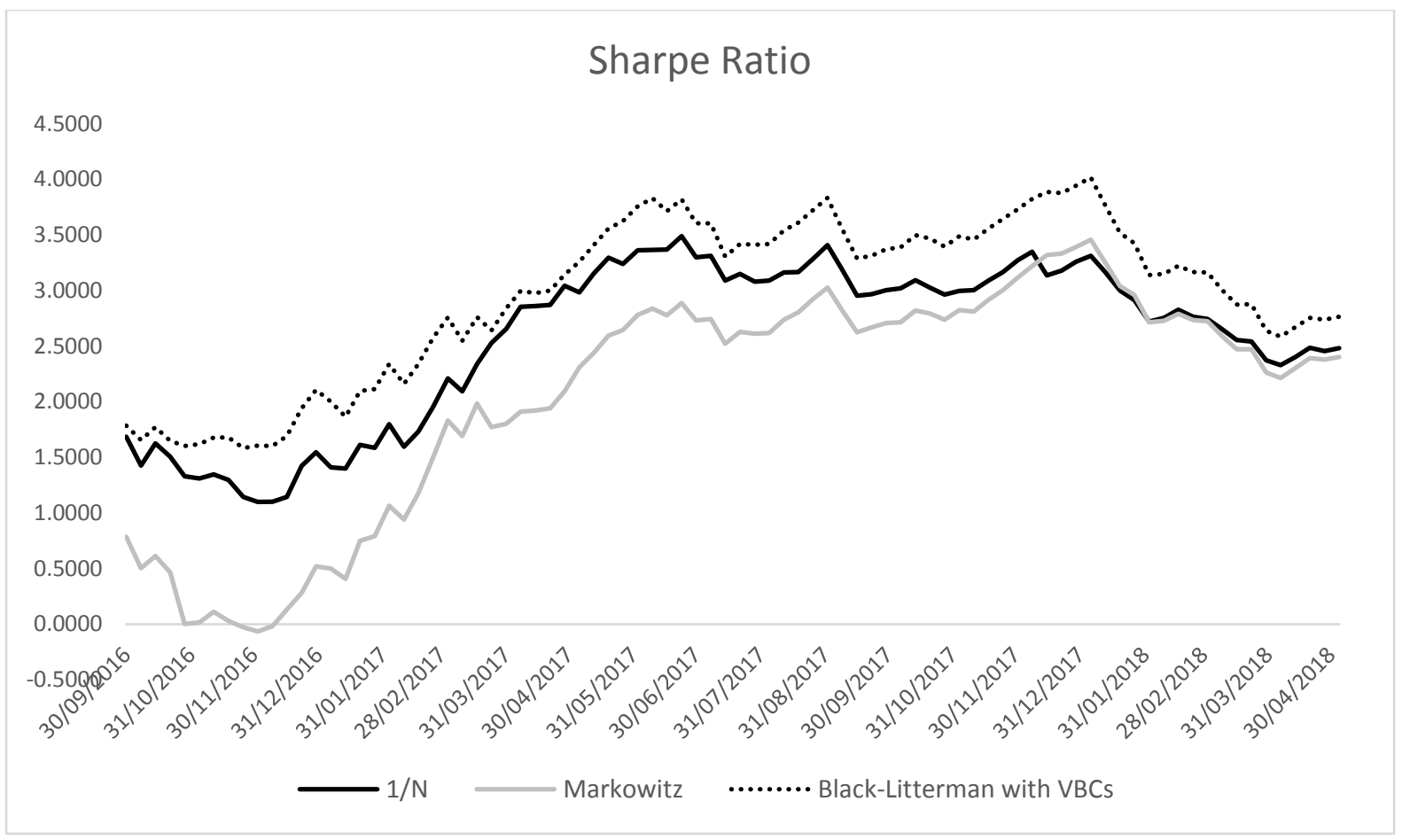

Figure 4: The out-of-sample Sharpe ratio over time of the advanced portfolio optimization model and the 2 benchmarks (1/N and Markowitz) by allowing for short-selling, inclusive of transaction costs, and for a 110-week rolling window.

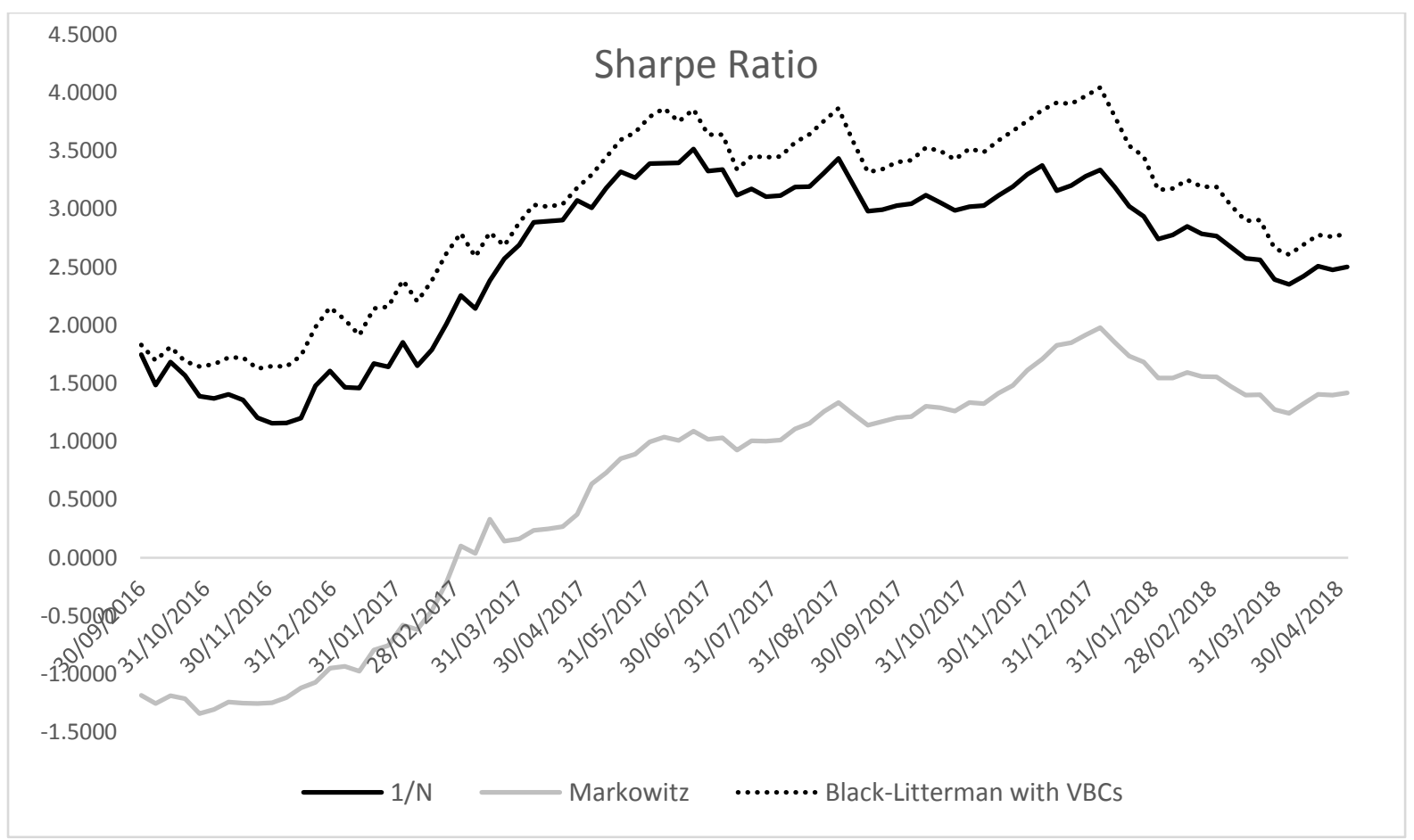




\section{References}

Baur, D. G., Hong, K., Lee, A. D. (2018). Bitcoin: Medium of exchange or speculative assets? Journal of International Financial Markets, Institutions and Money, 54, 177-189.

Bessler, W., Opfer H. and Wolff, D. (2017). Multi-Asset Portfolio Optimization and Out-of-Sample Performance: An Evaluation of Black-Litterman, Mean-Variance, and Naïve Diversification Approaches. The European Journal of Finance, 23, 1-30.

Black, F. and Litterman, R. (1992). Global Portfolio Optimization. Financial Analysts Journal, 48, 28-43.

Board, J.L.G. and Sutcliffe, C.M.S. (1994). Estimation Methods in Portfolio Selection and the Effectiveness of Short Sales Restrictions: UK Evidence. Management Science, 40, 516-534.

Bouri, E., Molnár, P., Azzi, G., Roubaud, D., Hagfors, L. I. (2017). On the hedge and safe haven properties of bitcoin: Is it really more than a diversifier? Finance Research Letters, 20, 192-198.

Chaim, P., Laurini, M. P. (2018). Volatility and return jumps in bitcoin. Economics Letters, 173, 158-163.

Corbet, S., Meegan, A., Larkin, C., Lucey, B., Yarovaya, L. (2018a). Exploring the dynamic relationships between cryptocurrencies and other financial assets. Economics Letters, 165, 28-34.

Corbet, S., Lucey, B., Urquhart, A., Yarovaya, L. (2018b). Cryptocurrencies as a financial asset: A systematic analysis. International Review of Financial Analysis, forthcoming.

DeMiguel, V. and Garlappi, L. and Uppal, R. (2009). Optimal Versus Naive Diversification: How Inefficient is the 1/N Portfolio Strategy? Review of Financial Studies, 22, 1915-1953.

Kajtazi, A., Moro, A. (2018). The role of bitcoin in well diversified portfolios A comparative global study. International Review of Financial Analysis, forthcoming.

Kan, R. and Zhou, G. (2007). Optimal Portfolio Choice with Parameter Uncertainty. Journal of Financial and Quantitative Analysis, 42, 621-656.

Levy, H. and Levy, M. (2014). The Benefits of Differential Variance-Based Constraints in Portfolio Optimization. The European Journal of Operational Research, 234, 372-381.

Lintilhac, P. S., Tourin, A. (2017). Model-based pairs trading in the bitcoin market. Quantitative Finance, 17(5), 703-716.

Markowitz, H. (1952). Portfolio Selection. The Journal of Finance, 7, 77-91.

Meucci, A. (2010). The Black-Litterman Approach: Original Model and Extensions. The Encyclopedia of Quantitative Finance, Wiley.

Oikonomou, I., Platanakis, E. and Sutcliffe, C. (2018). Socially Responsible Investment Portfolios: Does the Optimization Process Matter? The British Accounting Review, vol. 50, no. 4, pp. 379-401.

Platanakis, E. and Sutcliffe, C. (2017) Asset-Liability Modelling and Pension Schemes: The Application of Robust Optimization to USS. The European Journal of Finance, 23, 324-352.

Platanakis, E., Sutcliffe, C. and Urquhart, A. (2018a). Optimal vs Naïve Diversification in Cryptocurrencies, Economics Letters, 171, 93-96.

Platanakis, E., Sakkas, A., Sutcliffe, C. (2018b). Harmful Diversification: Evidence from Alternative Investments. The British Accounting Review (forthcoming).

Platanakis, E., Urquhart, A. (2018). Should Investors Include Bitcoin in Their Portfolios? A Portfolio Theory Approach. Available at SSRN: http://ssrn.com/abstract=3215321

Satchell, S. and Scowcroft, A. (2000). A Demystification of the Black-Litterman Model: Managing Quantitative and Traditional Portfolio Construction. Journal of Asset Management, 2, 138-150. 